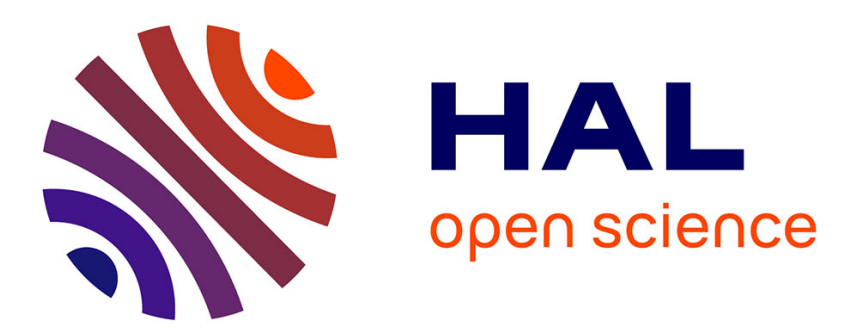

\title{
Cognitive Based Decision Support for Water Management and Catchment Regulation
}

\author{
Ioan Petri, Baris Yuce, Alan Kwan, Yacine Rezgui
}

\section{To cite this version:}

Ioan Petri, Baris Yuce, Alan Kwan, Yacine Rezgui. Cognitive Based Decision Support for Water Management and Catchment Regulation. 19th Working Conference on Virtual Enterprises (PRO-VE), Sep 2018, Cardiff, United Kingdom. pp.467-477, 10.1007/978-3-319-99127-6_40 . hal-02191166

\section{HAL Id: hal-02191166 https://hal.inria.fr/hal-02191166}

Submitted on 24 Jul 2019

HAL is a multi-disciplinary open access archive for the deposit and dissemination of scientific research documents, whether they are published or not. The documents may come from teaching and research institutions in France or abroad, or from public or private research centers.
L'archive ouverte pluridisciplinaire HAL, est destinée au dépôt et à la diffusion de documents scientifiques de niveau recherche, publiés ou non, émanant des établissements d'enseignement et de recherche français ou étrangers, des laboratoires publics ou privés. 


\title{
Cognitive Based Decision Support for Water Management and Catchment Regulation
}

\author{
Ioan Petri ${ }^{1}$, Baris Yuce $^{2}$,Alan Kwan ${ }^{1}$, Yacine Rezgui ${ }^{1}$, \\ ${ }^{1}$ School of Engineering, Cardiff University \\ Cardiff, Wales, UK \\ \{petrii,kwan,rezguiy\}@cardiff.ac.uk \\ ${ }^{2}$ College of Engineering, Mathematics and Physical Sciences, University of Exeter \\ Exeter, UK \\ b.yuce@exeter.ac.uk
}

\begin{abstract}
The effect of climate change on water ecosystems include increased winter precipitation, severe floods, leading to fluctuations in stream flow in areas and affecting both fish survival and water supplies. Several methods exist for establishing projections of changes in precipitation with regards to river flows and water levels at the river-basin scale, but hydrological characteristics change remain difficult to predict. Ensuring optimization techniques for water systems becomes significantly important especially with the degradation of water ecosystems and increased risks for fish population.

On the other hand, water demand has increased in the recent periods with the population growth. Further changes in the irrigation water system demand are determined by climate change precluding the reliability of current water management systems and affecting on the water-related ecosystems.

To address these challenges real time water management and optimization strategies are required to facilitate a more autonomous management process that can address requirements for water demand, supply and ecosystem preservation.

We present a cognitive based decision system that performs river level prediction for water optimization and catchment regulation for preserving Usk reservoir ecosystem in South Wales. The research is conducted on the Usk reservoir in South Wales reservation that is seeking to preserve the ecosystem and for which we propose a more informed decision system for catchment regulation and water management. Our system provides five days river level prediction to regulate river levels by pumping from/to reservoirs and to create artificial spates during the salmon migration season and to coincide with periods of low river flow.
\end{abstract}

Keywords: Decision support, Cognitive systems, River prediction, Catchment management, Ecosystem preservation.

\section{Introduction}

Research in water ecosystems has determined the need to use existing river catchment water supplies in a smarter way by having the ability to make decisions in real time. The objective is to address the decision making process involved in water management and catchment regulation by utilizing computer techniques and data 
analysis. This represents a key advantage for water resource coordination and process optimization by employing data mining techniques on various sources of data. As data is continuously increasing, with sensors and meters broadcasting real time readings it is possible to devise scenario based optimization, where several hypothesis at the catchment level can be tested, results can be analyzed and understood and a decision can be determined in relation to requirements and constraints of stakeholders [1], [2].

There is a growing research interest on water management and ecosystem preservation that has been triggered by the changing conditions in climate, new urban strategies and increase in population. Such factors have led to the development of new businesses and technologies in the field of water management for addressing the complexity in the workflows associated with water and catchment regulation. Research has reported over time, variability and increased parameters for water demand and consumption which requires specialized methods for conducting optimization and informed decision at a catchment level. The field of water optimization and catchment regulation is large and complex; therefore regulation strategies and optimization are needed in order to address the water supply and demand requirements but also to address the degradation of the water ecosystems.

Cognitive systems have been intensively used for multi-objective optimization problems for facilitation analysis of different parameters and variables that may have an impact on a modelled phenomenon. Such systems require a data capture process from sensors and meters but also an expert computing infrastructure for conducting analysis [3], [4]. With such cognitive systems several interactions between the different sensors, devices, actuators, and controllers are facilitated, leading to the creation of "intelligent" collaborative decision systems which have embedded monitoring and controlling equipment and the potential to optimize various objectives along with operations and maintenance expenses [5], [6].

Cognitive based decision systems represent methods that use language processing mechanisms and machine learning techniques to simulate an existing reality and to assess potential risks that may be triggered in relation to monitoring parameters and studied objectives. Cognitive systems can be trained based on historical data by employing artificial intelligence algorithms and have the ability to mimic an existing system process and can facilitate behaviors, reasoning, adaptive reactions and an overall autonomy.

In this paper we present a prediction based cognitive system for decision support in water management and catchment regulation. The solution we propose is based on scenario modelling with artificial intelligence techniques for providing process management tools and to support informed decisions for users involved in the process on water resource preservation and regulation. Our system provides five days river level prediction to regulate river levels by pumping from/to reservoirs and to create artificial spates during the salmon migration season and to coincide with periods of low river flow. The described system supports collaboration of stakeholders in water management and facilitates integration of different knowledge systems greatly addressing the problem of catchment regulation.

The rest of the paper is organized as follows: In Section 2 we conduct a review of the state of the art from the field of water research; in Section 3 we present the approach and methodology of this study. We present the prediction system in Section 4 and we provide results and conclusions in Section 5, and Section 6. 


\section{Related Work and Approach}

The recent research on water ecosystems and environment preservation aims at achieving a step change in water savings by applying and testing intelligent techniques for real time abstraction and discharge monitoring. There is an increased interest in creating an open, scalable, marketable and user-friendly system to optimize water resource management and replace the current licensing system. An innovative "just-in time" ability is required for creating substantial water savings, whilst also enhancing delivery on EU Water Framework Directive obligations. The objective on these researching attempts is to combine real time traditional catchment data with remote sensing to provide greater spatial assessment of catchment hydrological variability. Data systems development, predictive modeling and analysis are required in order to undertake and to test in catchments with farming, industrial and ecological stakeholders; and the regulatory viability developed with Natural Resources Wales.

The analysis presented in this paper are applied on the Usk river that has smaller abstractions for recreation and agriculture, but the test bed has been shown to be a robust model for constructing and testing scenario-based optimization. The mean annual daily water demand is around $200 \mathrm{Ml}$ /day from the Usk where the River Usk also provides an important site for testing freshwater ecological requirements. Natural Resources Wales collaborate with the Usk and Wye Abstractors Group (and other organizations) to define the ecological flow requirements for ecosystem preservation. Although the Usk catchment is dominated by surface water supplies, groundwater plays a significant part in the water supply through boreholes - e.g at Brecon. Groundwater factors needs to be considers and assessed when create a decision model. Transferability to other catchments and expansion to model conjunctive use schemes, such as SEWCUS (South East Wales Conjunctive Use System) are objectives to be addressed in order to achieve a smarter water management.

Several research studies on water optimization are seeking to devise a more intelligent water management system by conducting dependency analysis and modelling for water resources and corresponding mechanisms for adaptation, demand and response to changes. The results of these studies have provide useful insights for the water management domain and delivered an improved quality-of-services to respond to continuous increase of water demand. This study aims at designing and implementing a cognitive based system for water resources but also inform the work on water distribution networks where new parameters are relevant such as: topology, pressure control, leakage and resilience. The realisation of an intelligent system for water management should therefore take into consideration concepts from ICT, cloud and big data all connecting to data capture points supported with sensing capability and prediction in water level to facility water network management and regulation [7]. 


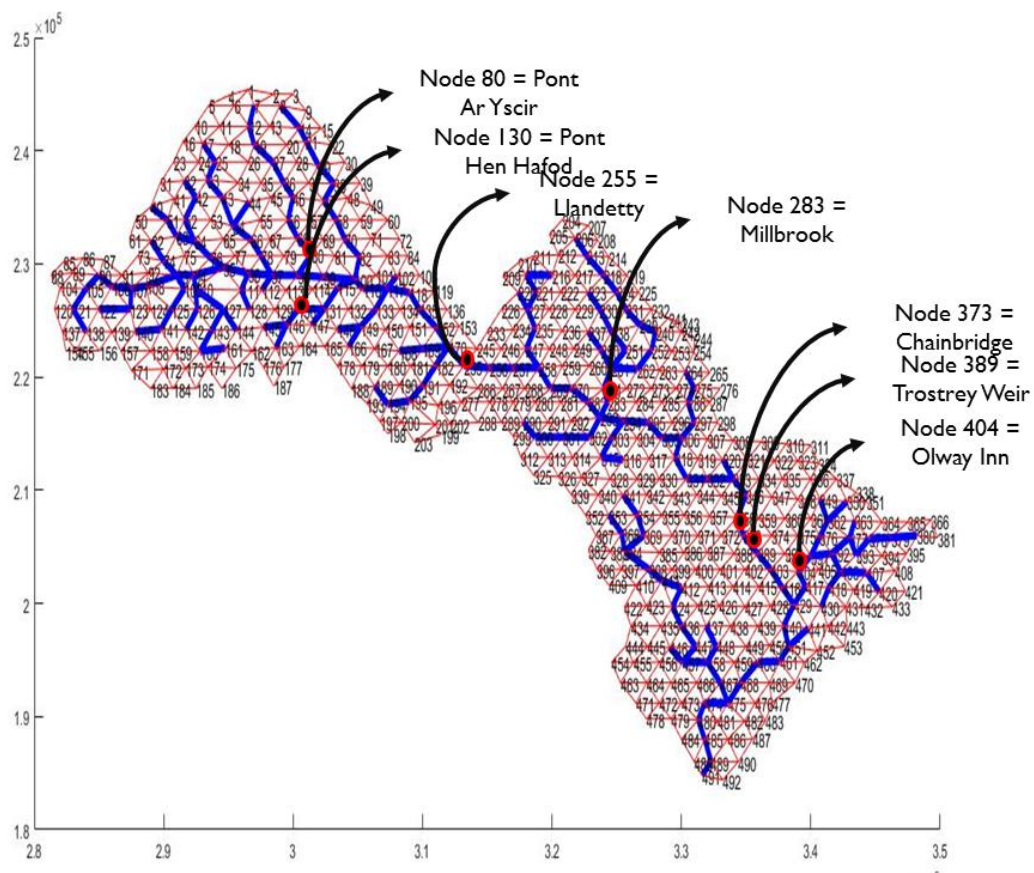

Fig.1. The Usk Reservoir model: Nodes identify stations that are part of our analysis: Pont ArYscir, Pont Hen Hafod, Llandetty, Millbrook, Chainbridge, Trostrey Weir, Olway Inn.

Prediction based cognitive systems use artificial neural networks for rainfall probability modelling and represents a key strategy to serve in the process of planning, utilisation and cording of water based workflows [8]. There are multiple data analysis and mining tools and techniques leveraging on artificial neural networks aiming at providing water projections and forecasting of events which have an associated probability of occurrence [9], [10]. Similar techniques are Support Vector Machine (SVM) techniques, that are frequently used for undertaking hydrological modelling and address the increased complexity that is derived with the modelling and prediction process for water management [11], [12].

In this paper we present a prediction based cognitive system for river catchment regulation to assist users and stakeholder to collaborate in the process of decision management. We use the Usk reservoir (see Figure 1) as a trial case utilising raw data captured from water stations sensing equipment and employ artificial neural networks techniques to forecast river level and flow for the stations part of the trial project. This cognitive system is then aimed to serve for a water decision system that can support actors involved in water business and ecosystem preservation to manage risk and conduct real time analysis for catchment regulation. 


\section{Methodology}

The decision support cognitive system proposed in the paper utilizes artificial neural techniques to deliver five days forecasting in river depth and river flow for the Usk reservoir. We devise our system in multiple layers which interact and exchange data with the general objective of facilitating water management and ecosystem preservation.

\subsection{Architecture}

The architecture of the system is formed by three different layers which interact and facilitate data transfer and transformation from data capture layer to user decision support:

In the data capture layer, data is retrieved from the capture points identifying the seven stations within the Usk river. We have implemented a service that retrieves data based on hourly basis for the following stations:" PontArYscir", "Pont Hen Hafod", "Llandetty", "Millbrook", "Chain bridge", "Trostrey Weir", "Olway Inn".

The reading points at the station level broadcast periodical river depth values which are then transformed into equivalents river flow values by the prediction layer. To conduct the cognitive process weather data is also retrieved by a service that stores rainfall information associated with the reservoir from the UK Met Office weather service.

For the prediction layer we have implemented several modules: the Harbour module for station river data sets, the neural network module for prediction and the iDepend module for dependency modeling module.

In the Harbour module, developed by Cambrensis (an industrial partner), we store the river depth datasets which is further used as an input for the prediction module. This module is formed of services that retrieve river data via an HTTP layer triggered on hourly intervals.

The artificial neural network (ANN) utilizes three main prediction objectives: river depth, river flow and rainfall. These objectives support the overall forecasting at the catchment level and are used to create a more informed decision making system for users.

The i-Depend module enables a dependency modeling process and utilizes as inputs the results generated by the prediction model. An interaction between layers and modules alongside the overall architecture of the system are presented in Figure 3. To support the functionality of the system for data analysis, forecasting and dependency modeling, a cluster based infrastructure is used. 


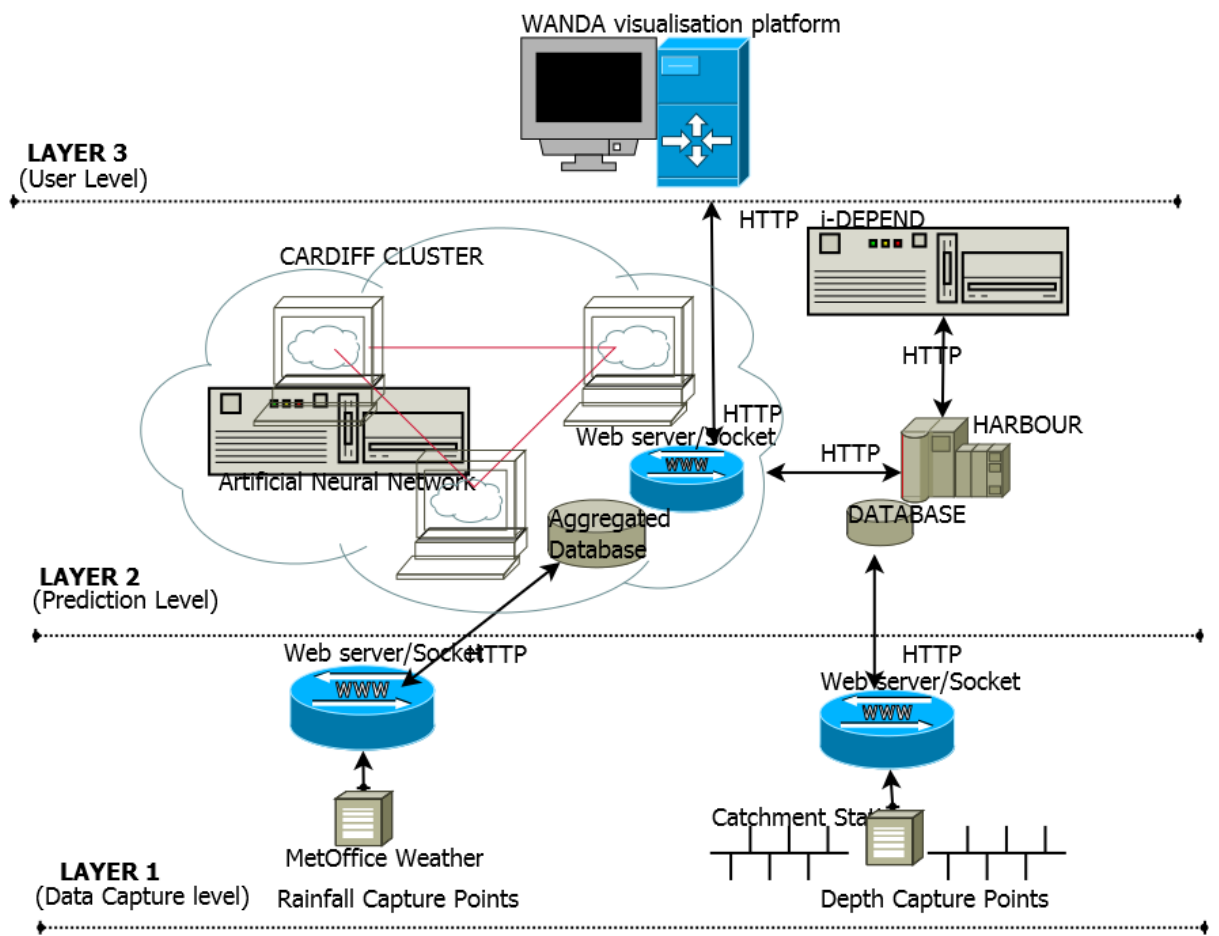

Fig. 2. The cognitive based decision support system architecture; The system includes: (i) the data capture layers used to fetch river data, (ii) the artificial neural network layer used to predict river flow and river depth and (iii) the user layer for presenting the results of the prediction for decision makers.

The user layer facilitates direct interaction between users and the computer based system where several predictions and dependency modeling techniques are undertaken in relation to scenario data analysis for the catchment regulation. The web interface takes into account users profiles and preferences and enables users to access knowledge representation based on the previous analysis.

Users can create specialized use-cases based on agreed objectives to understand how the reservoir can evolve and to simulate river scenario projections that can inform decision and mitigate possible risks. 


\subsection{Modeling the Cognitive System with Artificial Neural Network}

For the cognitive system we consider an ANN (Artificial Neural Network) prediction function with inputs and pre-determined objectives such as: $\mathrm{f}$ (a) $: \mathrm{Ia} \rightarrow \mathrm{Ra}$, where Ia : [di , ri ] represents the input of the ANN system (di is the river depth of node $n_{i}$, where node $n_{i}$ is a station $s_{i}$ from the reservoir), $r_{i}$ identified the rainfall of node ni and $\mathrm{Ra} \rightarrow\left[\mathrm{p}_{\mathrm{d}}, \mathrm{p}_{\mathrm{f}}, \mathrm{p}_{\mathrm{r}}\right]$ represents the results associated with the prediction engine ( $\mathrm{p}_{\mathrm{d}}$ is the predicted depth for $n_{i}$, pf is the predicted flow for $n_{i}, p_{r}$ is the predicted rainfall). The set of input values $I_{a}$ is formed of readings recorded at the station level whereas $R_{a}$ determines the output for the prediction engine identifying river depth, river flow and rainfall.

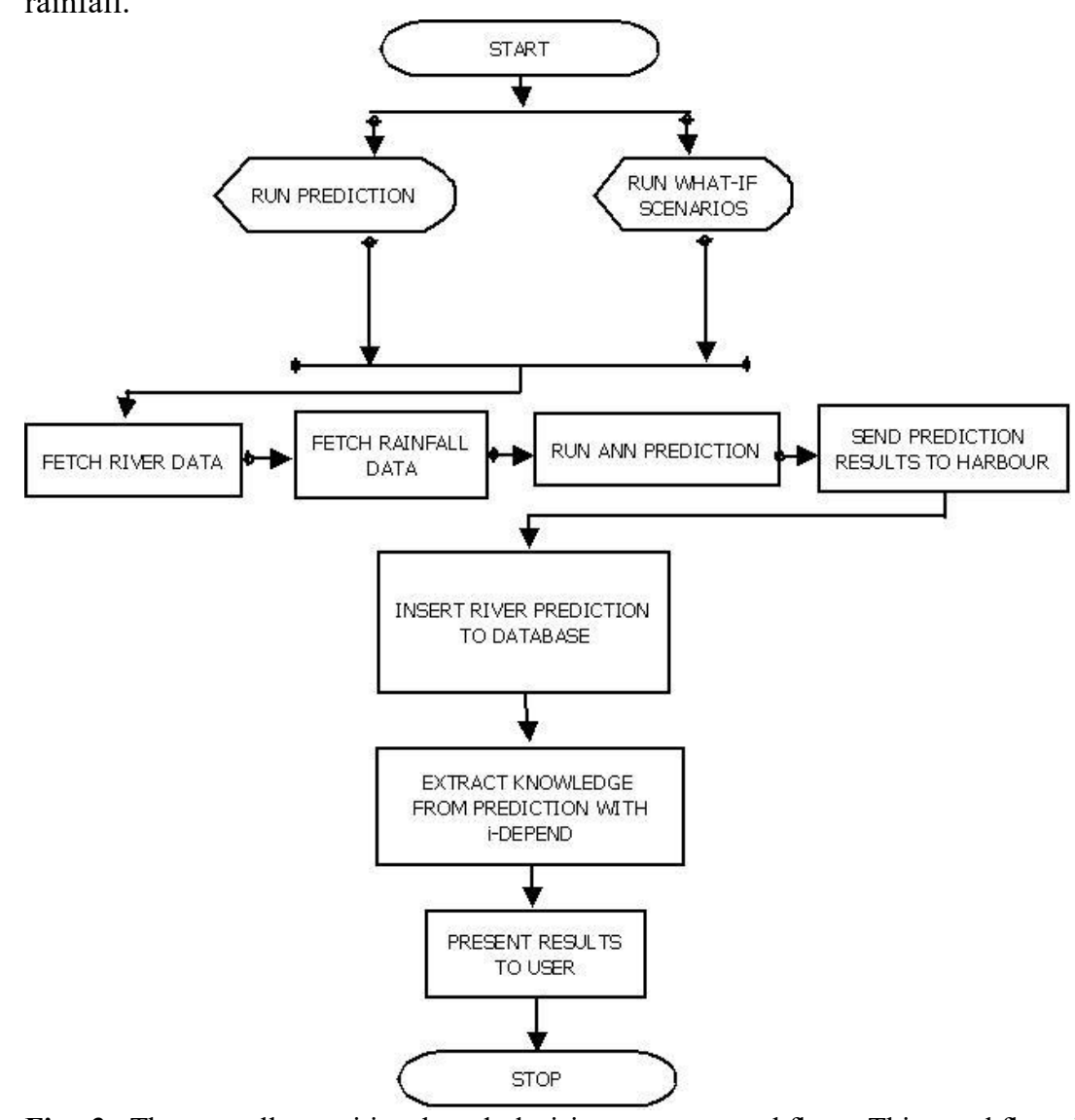

Fig. 3. The overall cognitive based decision system workflow: This workflow identifies: scenario definition phase to support the prediction, data capture phase with (i) river data and (ii) rainfall data, prediction deployment and user interfacing with results. 
The development of the ANN system identifies several stages in relation to training and calibration. The artificial neural network involves calibration stages where a cost function is utilized for increasing the accuracy of the forecasting process. The training is performed over multiple rounds and multiple historical datasets which are prepared and pre-processed to serve into the prediction process. The ANN has been configured to return the river depth, river flow and rainfall for the sever stations within the Usk reservoir as illustrated in Figure 3.

Several ANN models have been tested to find the best configuration on both Visual Studio platform and MATLAB. For C++ Based Fast ANN (FANN) models we have used:(i) Standard Backpropagation - where the weights are updated after each training pattern and (ii) Advanced batch training - not use the learning rate (default training algorithm). For MATLAB based ANN models we have used: (i) Conjugate Gradient Backpropagation with Powell-Beale restarts and (ii) Gradient Descent Backpropagation.

The elaboration of the ANN module consisted in multiple training rounds where $80 \%$ of the stored datasets have been utilized while the remaining $20 \%$ was utilized for the testing phase. With the training and calibration process the results accuracy of the ANN has been identified in value of $93 \%$ for all the selected objectives: river depth, river flow and rainfall. A challenge in the ANN development process was the quality of data and fluctuation of the recorded valuess to which we have applied different normalization techniques which led to an improvement in the overall results. In total the resulted ANN performance for one step ahead prediction of river depth, river flow has been indentified in average of $93 \%$. An exception is the rainfall which has been recorded with an accuracy of $89 \%$.

\section{Evaluation}

In this section we test several scenarios in order to validate the efficiency of our cognitive based decision system. The overall objective is to generate accurate predictions which will serve the user in the process of decision support with forecast on river depth for a period of five days in advance. Scenario 1 illustrates the input phase on the cognitive system where real data values are fetched from the reservoir and inserted into the prediction process (see Figure 4) followed by the predicted output identifying predicted river depth for a period of five days per station (see Figure 5). Scenario 2 presents five days historical trend and five days predicted trends as resulted from the system (see Figure 6).

\subsection{Scenario 1: Overall Catchment River Level Prediction}

This scenario investigates the impact of river depth as a mean to monitor the evolution of the Usk reservoir in the determined evaluation interval of five days. With data retrieved from the capture points at the station level and rainfall recorded with a services dedicated for the MetOffice UK, the system determines trends for river depth for an interval of five days. 
As reported in Figure 4, there are several variables that we consider in the overall scenario development phase which after rounds of modeling, training and forecasting can provided the evolution of river depth for the monitored stations. In Figure 5 we present the results of the scenario reported in Figure 4, with predicted values for all stations and a graphical comparison of the predicted river depth and actual river depth.

It can be observed that the cognitive system is based on the ANN engine and can support simulation of various what-if scenarios where possible events at the river level and at the weather level can be identified and impact can be determined for a period of five days in future. Such scenario based simulation can prove to be extremely efficient for users involved in the process of decision management at the river level and can lead to more informed strategies and coordination of a water ecosystem.

River data fetched from Harbour

\begin{tabular}{|c|c|}
\hline Time: & $12: 13: 42$ \\
\hline Date: & 2017-01-10 \\
\hline Rainfall Per Day $\left(\mathrm{mm} / \mathrm{m}^{\wedge} 2\right)$ : & 31.0 \\
\hline River Depth Station "Pont Ar Yscir" $(m / m \wedge 2)$ : & 0.26 \\
\hline River Depth Station "Pont Hen Hafod"( $\left.\mathrm{m} / \mathrm{m}^{\wedge} 2\right)$ : & 0.408 \\
\hline River Depth Station "Llandetty" $\left(\mathrm{m} / \mathrm{m}^{\wedge} 2\right)$ : & 0.755 \\
\hline River Depth Station "Millbrook" $\left(\mathrm{m} / \mathrm{m}^{\wedge} 2\right)$ : & 0.131 \\
\hline River Depth Station "Chainbridge" $\left(\mathrm{m} / \mathrm{m}^{\wedge} 2\right)$ : & 0.682 \\
\hline River Depth Station "Trostrey Weir" $\left(\mathrm{m} / \mathrm{m}^{\wedge} 2\right)$ : & 0.394 \\
\hline River Depth Station "Olway Inn"( $\left.\mathrm{m} / \mathrm{m}^{\wedge} 2\right)$ : & 0.252 \\
\hline Usk Reservoir (\%) & 50 \\
\hline Talybont Reservoir (\%) & 60 \\
\hline Llandegfedd Reservoir (\%) & 55 \\
\hline
\end{tabular}

Fig. 4. The data capture phase within the prediction based cognitive process: Several scenario variables are retrieved and used as input for the prediction phase. These variables are of two types: (i) river based variables and (ii) system based variables.

Figure 4 illustrates the input of the simulation where variables have been selected after sensitive analysis of the overall repository of historical data. These historical data have informed the modeling process of the ANN which has returned the forecasted values for station river depth as illustrated in Figure 5. 


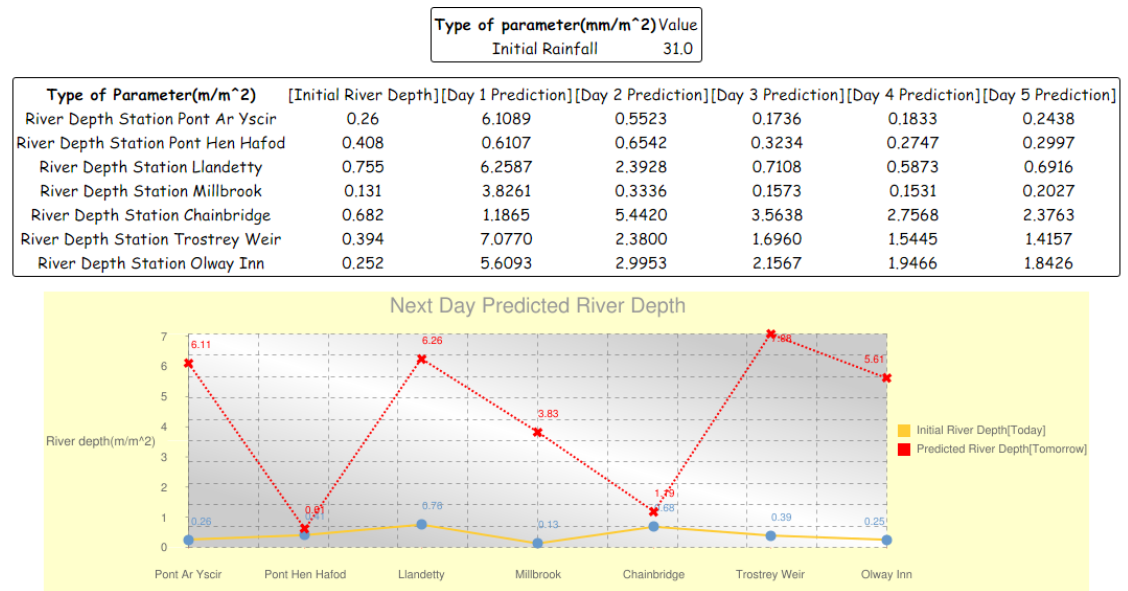

Fig. 5. The output of the decision process; identifying initial river depth and initial rainfall together with the predicted values for river depth, on five days forecast interval for all seven stations within the Usk Reservoir.

\subsection{Scenario 2: Station River Depth Prediction}

This scenario focuses on per station evaluation of the river depth in order to provide a more concise and focused evaluation. On a station level the user is more interested in determining shorted intervals evolution of the river depth in relation to variables that can impact such river depth projections independently to other stations. Using a similar methodology as in the multiple stations context, Figure 6 illustrates past, current and future river depth trends for river depth and rainfall on a per station basis. The analysis are conducted on the intervals [Day -5, Days 5], where [Day 0] is today, [Day -5] represents five days ago, [Day 5] represents five days ahead. From the experiments, it can be identified the similarity in fluctuation between river depth and rainfall leading to the conclusion that a change in the rainfall at the reservoir level has a direct impact of the river level at the station level. This can be utilized by actors involved in the process of decision making by proceeding to release or retain water from/in the reservoir based on specific water management strategies. 


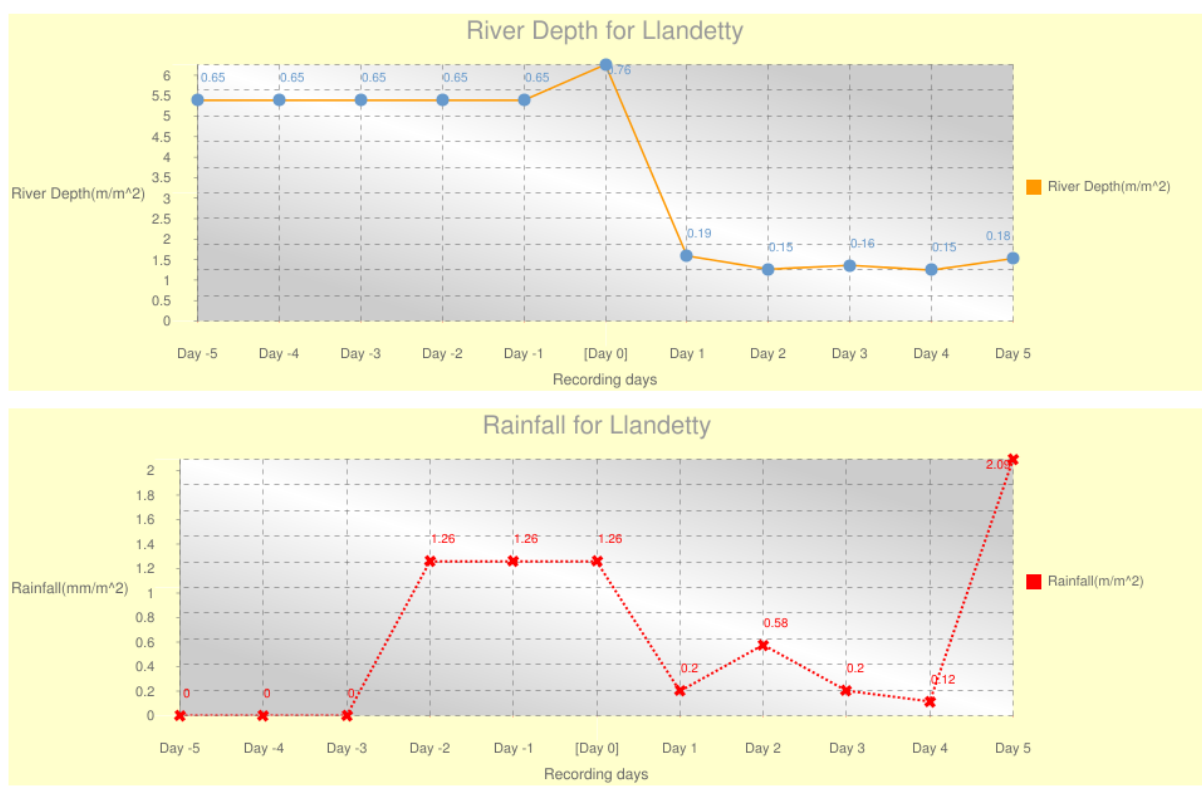

Fig. 6. Station based prediction with historical river depth and rainfall: In this illustration Llandetty station is presented for five days historical and five days forecast. [Day 0] represents the current day whereas [Day -5, Day -1] is a five days historical period and [Day 1, Day 5] represents five days in future.

\section{Conclusions}

Water security and climate change are major challenges, now and for the future and where more and more research is concluding that many solutions will be complex and require a holistic approach. In this paper we have established a cognitive based decision support system that provides the necessary tool that can cut through some of this complexity and help users to test and evaluate scenarios that will help in the understanding and visualization of a water related event. Our solution can open up new opportunities to provide additional decision services to existing customers/ clients as well as promoting and using this new and enhanced expertise on water management to new prospects.

In this study we present a cognitive based decision system engine to support water simulation and prediction. The initial analysis is undertaken at a per-station level to understand the impact of rainfall at the level of a station and to study various relevant scenarios which can inform the implementation of strategic decisions at the catchment level. The Usk reservoir has represented an extremely dynamic pilot project where multiple variables are involved and multiple objectives need to be assessed in order to create a more informed water management process based on a cognitive evaluation supported by ANN algorithms. Such development at the catchment level can be of 
impact to manage water resources and to address the set of factors associated with the process of water management and catchment regulation.

Acknowledgments. This work is part of the Innovate UK Water Security Project: "Developing a Real Time Abstraction \& Discharge Permitting Process for Catchment Regulation and Optimized Water Management.”, grant number: 504460

\section{References}

1. McCann, Julie A., et al., "Optimized Water Demand Management Through Intelligent Sensing And Analytics: The WISDOM Approach." (2014).

2. Singh et al, Appropriate data normalization range for daily river flow forecasting using an artificial neural networks, Hydroinformatics in Hydrology, Hydrogeology and Water Resources. International Association of Hydrological Sciences Publication, Uk, pp. 51-57, 2009

3. Ioan Petri, Javier Diaz-Montes, Mengsong Zou, Ali Reza Zamani, Tom Beach, Distributed Multi-Cloud Based Building Data Analytics, Kecskemeti, Gabor, Attila Kertesz, and Zsolt Nemeth. "Developing Interoperable and Federated Cloud Architecture." IGI Global, 2016. 1-398. Web. 24 Feb. 2017.doi:10.4018/978-1-5225-0153-4

4. BarisYuce, Haijiang Li, Yacine Rezgui, Ioan Petri, BejayJayan, Chunfeng Yang, Utilizing artificial neural network to predict energy consumption and thermal comfort level: An indoor swimming pool case study, Energy and Buildings, Volume 80, September 2014, Pages 45-56, ISSN 0378-7788.

5. T. Robles et al., "An Internet of Things-Based Model for Smart Water Management," Advanced Information Networking and Applications Workshops (WAINA), 2014 28th International Conference on, Victoria, $\mathrm{BC}, 2014$, pp. 821-826, doi: 10.1109/WAINA.2014.129

6. KUMURA Takahiro, SUZUKI Naofumi, TAKAHASHI Masatake, TOMINAGA Shin, MORIOKA Sumio, Ivan Stoianov, Smart Water Management Technology with Intelligent Sensing and ICT for the Integrated Water Systems, Special Issue on Solutions for Society Creating a Safer and More Secure Society, January, 2015.

7. Hideyuki Tadokoro, Makoto Onishi, Koji Kageyama, HiromitsuKurisu, Shinsuke Takahashi, Smart Water Management and Usage Systems for Society and Environment, Hitachi Report,pp. 264-174, 2016.

8. P.S. Dimitri, N.D. Khada, Model trees as an alternative to neural networks in rainfall-runoff modelling, Hydrological Sciences Journal 48 (3) (2003),399-411.

9. V. Vapnik, The nature of statistical learning theory, New York: Springer-Verlag, 1995.

10. Schlkopf B., Smola A., Williamson R. and Bartlett P., New Support Vector Algorithms, Neural Computation 12 (2000) 1207-1245.

11. Y. Liu, G. Pender, Carlisle 2005 urban flood event simulation using cellular automatabased rapid flood spreading model, Soft Computing, 17(1) (2013) 29-37.

12. W. C. Wang, K. W. Chau, C. T. Cheng, L. Qiu, A Comparison of Performance of Several Artificial Intelligence Methods for Forecasting Monthly Discharge Time Series, Journal of Hydrology 374(3-4) (2009) 294-306. 